\title{
Identifying pedagogical intervention in MOOCs learning processes: a conversational agent proposal
}

\author{
Diego Rossi ${ }^{1}$, Victor Ströele ${ }^{1}$, Fernanda Campos ${ }^{1}$, Regina Braga $^{1}$, José Maria N. David $^{1}$ \\ ${ }^{1}$ Universidade Federal de Juiz de Fora (UFJF) \\ Programa de Pós-graduação em Ciência da Computação \\ Rua José Lourenço Kelmer, s/n - Campus Universitário - 36036-900 - Juiz de Fora/MG
}

\begin{abstract}
Monitoring students in virtual learning environments can be a timeconsuming task. Professors and tutors must accompany students in an agile manner. During the COVID-19 pandemic, the use of discussion forums posed new challenges. This work proposes a conversational agent to automatically detect which pedagogical intervention is necessary to guide students in MOOCs environments. Through the attributes of the students' post messages, it is possible to classify which action will be carried out by the agent, applying specific dialogue patterns. In some more specific cases, the tutor's attention is immediately requested. The proposal was evaluated through a feasibility study to verify if semantic detection can contribute to guide the intervention process. According to the results, it is possible to support the tutor, as only $35.2 \%$ of interactions required the tutor's action.
\end{abstract}

\section{Introduction}

The popularization and advances in the area of information technology made it increasingly part of our daily lives. Its use ended up indispensable for the educational environment, including supporting pedagogical practices. Enabling people to study at a distance democratizes access to education, with a more attractive price, and allowing them to set up their study schedules and have full-time jobs. The institution that works in this type of education has a leaner structure, generating savings with classrooms, laboratories, etc.

With the COVID-19 pandemic, new challenges are posed considering remote learning: technologies, evasion, distancing, students and teachers preparation, monitoring the evolution of students, among others. These challenges demand that professors and tutors accompany students in an agile manner, providing a communication environment capable of answering questions and motivating students. With that, we believe that assisted education may contribute to the automatization process of students tutoring. In this context, the greatest challenge is not only sending students recommendations and academic topics but also predicting learning issues and sending notifications [Toti et al. 2020].

Discussion forums are among the most popular interaction tools offered by MOOCs, often used by students to create a sense of belonging and better understand course topics [Capuano and Caballé 2015]. However, students trying to clarify concepts through these forums may not get the attention they need, and the lack of responsiveness often favors drop-out [Capuano and Caballé 2019]. Thus, the semantic detection of forum posts offers implicit information, which requires a more careful analysis to obtain an assessment of the student's knowledge, intending to moderate and plan interventions. 
A conversational agent (CA) is an artificial intelligence (AI) entity usually operating based on a well-defined set of rules that shape its behavior when interacting with humans by imitating human conversations through voice commands, text chats, or both [Demetriadis et al. 2021]. Researchers have already experimented using conversational agents to accomplish various educational goals such as tutoring, question-answering, language learning practice, and the development of metacognitive skills.

We are currently witnessing a significant advance in natural language processing with the popularization of voice assistants. These devices bring a conversational agent with a more spontaneous way of interaction [Callaghan et al. 2018]. This dialogue with a computer system is also increasingly present in educational environments. So, agents' actions are in search of more meaningful interactions, whether with instructors or peers.

This paper presents the Conversational Agent for Educational Recommendation System - CAERS. It is part of a project that aims at carrying out detection and analysis of the students' engagement within the context of online higher education and the e-learning platforms, avoiding school dropout [Capuano and Caballé 2019][Bóbó et al. 2019][Braz et al. 2019]. It combines Semantic Analysis (SA), and Natural Language Processing (NLP) approaches applied to Text Categorization (TC) of the forum posts and assignments from the students, combined with ML-based classification techniques. Our proposal is to integrate a conversational agent service in MOOCs to facilitate peer dialogue during chat-based activities.

The identification of the semantic patterns in the messages was built up from earlier defined approaches [Capuano and Caballé 2019, Bóbó et al. 2019, Braz et al. 2019]. This work aims to identify the pedagogical intervention necessary to guide the student in the learning process through the semantic patterns present in the students' posts in MOOCs. Through the attributes of the students' post messages, it is possible to classify which action will be carried out by the agent, applying specific dialogue patterns. Sentiment, type of post, urgency, and confusion [Capuano and Caballe 2019] are the attributes that provide the basis for the agent intervention in the student's learning process.

The main research question is: Is it possible to help the tutor to monitor the students' posts, by automatically detecting the pedagogical intervention through semantic rules? The proposed solution consists of storing the students' posts and their attributes in an ontology, making it possible to make inferences to detect the necessary pedagogical intervention, guiding the conversational agent.

The study followed three main steps: (i) definition of a semantic pattern to define the style of intervention in the pedagogical process; (ii) validate the semantic model for choosing the style of approach for the agent pedagogical intervention; (iii) evaluate the approach in a real education context of a distance education course, using MOOC students' posts. The proposal was evaluated through a feasibility study, where it was possible to verify if our solution can monitor the students' posts and the agent is able to take action automatically. In some more specific cases, the tutor's attention is immediately requested.

This article is structured as follows. Section 2 shows the related work. In Section 3 , the CAERS framework is presented, detailing the main components that make up the tool's workflow. A feasibility study is presented in Section 4, describing the educational scenario, dataset, and obtained results. In Section 5, the final remarks are presented. 


\section{Related Work}

Discussion forums in a Massive Open Course environment bring many challenges, including the significant amount of highly unstructured information, the number of students concerning the number of instructors, and the great effort to answer the posts. Otherwise, research systematically explores technology-enhanced learning solutions to increase students' engagement and avoid school dropout. Considering the research areas on which this paper draws, we selected articles addressing the automatic categorization of student forum posts [Capuano and Caballe 2019, Agrawal et al. 2015, Bóbó et al. 2019], autonomous agents applied to pedagogical interventions [Toti et al. 2020, Demetriadis et al. 2018], and agents associated with recommendation systems [Harrathi et al. 2017, Brigui-Chtioui et al. 2017, Bóbó et al. 2019].

[Capuano and Caballé 2019] use a very rich data source to tackle discussion forums in a MOOC environment. They proposed a multi-attribute text categorization tool able to automatically detect useful information from MOOC forum posts, including intents (question, answer, and opinion), topics covered, sentiment polarity, and level of confusion and urgency. The extracted information may be used directly by instructors for moderating and planning their interventions and input for conversational software agents able to engage learners in guided, constructive discussions through natural language.

[Agrawal et al. 2015] proposed YouEDU, which helps students, detecting all messages that express content confusion. Their work was the base for [Capuano and Caballé 2019] multi-attribute text categorization considering the confusion aspect. The authors trained a set of classifiers to categorize the forum posts in several aspects: sentiment, urgency, and other descriptive variables that guide a classifier to detect confusion. Then, the confusing posts are directed to video experts from the course.

The work developed in [Toti et al. 2020] aims to detect and analyze the involvement of course participants in the context of online education, obtaining relevant information related to aspects that indicate student engagement, such as sentiment, urgency, confusion, and the probability of student drop-out. Students' posts and comments are considered to accomplish this task, using classification algorithms based on machine learning.

[Bóbó et al. 2019] present the SASys architecture, based on a lexical approach and a polarized frame network. The main goal is to identify the author's sentiment in texts. The semantic orientation of the text is determined from the result of sentiment analysis approaches (Lexical, Machine Learning, or Hybrid). The author's emotional state begins with the data extraction from social networks, forms, or Virtual Learning Environments, then pre-processing techniques are applied to the texts. After that, they are submitted to the complex frame network for the identification of words with polarity. Three polarities were defined: positive - words that evoke a positive sentiment, negative - words that evoke a negative sentiment, and neutral - words that do not make a previous judgment. In the end, a recommendation system based on the student's emotional state and learning style sends motivational messages to mitigate dropout.

[Demetriadis et al. 2018] proposed the colMOOC platform, where a CA is modeled in a specific domain to mediate between dyads by triggering appropriate interventions to facilitate productive dialogue during chat-based collaborative learning activities in the massive education, such as MOOCs. The agent's interaction in the chat is based on the 
"Academically Productive Talk", a framework for modeling experienced teacher's interventions during students' dialogues to make them elaborate in the knowledge domain.

According to [Harrathi et al. 2017], students' dependence on poor assistance and adaptation in massive courses leads them to lose motivation and, consequently, to drop out of the learning process. As a solution, the authors developed a strategy for adapting activities through a hybrid recommendation system based on knowledge, supported by ontology, to recommend activities for students in the context of MOOCs.

[Brigui-Chtioui et al. 2017] proposed an agent-based recommendation system to help students overcome their disabilities. The system recommends resources to provide support to improve the learning experience. Also, an agent-based cooperative system was designed, where agents act independently and update recommendation data, making the recommender more efficient and enhancing experiences on the learning platform.

Our conversational agent is based on [Capuano and Caballé 2019, Agrawal et al. 2015] text categorization considering mainly the topics covered, level of confusion, and urgency. For the sentiment polarity categorization, we adopted [Bóbó et al. 2019, Demetriadis et al. 2018] approaches. As in [Toti et al. 2020, Bóbó et al. 2019] works, our research explores technologies such as knowledge representation, artificial intelligence, computational linguistic, among others, to increase students' engagement and avoid school dropout. As [Demetriadis et al. 2018], we also define conversational agents as software programs engaging learners in a conversation through natural language, hoping to impact group learning outcomes. We plan a highly innovative smart conversational agent chat-based service that may take place in a MOOC. Our proposal as in [Bóbó et al. 2019] will act in Virtual Learning Environments. A recommendation system, as proposed by [Harrathi et al. 2017], and also demonstrated in our group's previous works [Neves et al. 2020] [Bóbó et al. 2019], will be a component of CAERS project. Different from [Brigui-Chtioui et al. 2017] CAERS conversational agent is not recommending learning resources at this moment. However, as in [Bóbó et al. 2019], messages will guarantee peer dialogue with the students. Semantic detection is performed using messages posted in discussion forums, detecting the need for help, and assigning an action to the conversational agent in each situation, taking into account the parameters detected in the messages.

\section{CAERS Framework}

CAERS (Conversational Agent for Educational Recommender System) is a framework that detects the required pedagogical intervention when students post messages to forums in a MOOC environment. To this end, an ontology capable of determining the agent's actions and which educational intervention must be carried out is proposed. Highlighting CAERS key aspects and the functionality of the architecture we describe in Figure 1 its major components and workflow. The first step is to obtain the student's message. After this step, an annotation is performed, classifying the messages according to attributes considering the methodology defined in [Rossi et al. 2021], giving rise to a messages dataset. Then the records from that dataset are loaded into an ontology, and the semantic rules are applied to determine the best pedagogical intervention. Finally, the conversational agent automatically responds to the message, and, if necessary, it will contact other actors (tutor, class) to interact in the pedagogical intervention process. 
The messages used to carry out experiments were taken from Stanford MOOCPosts dataset. They have annotations performed manually, giving rise to the attributes used by the semantic rules [Agrawal et al. 2015]. During the development of this work, the main objective is to identify the most appropriate pedagogical intervention according to the student's post. Therefore, the tasks were concentrated on the knowledge and intervention components.

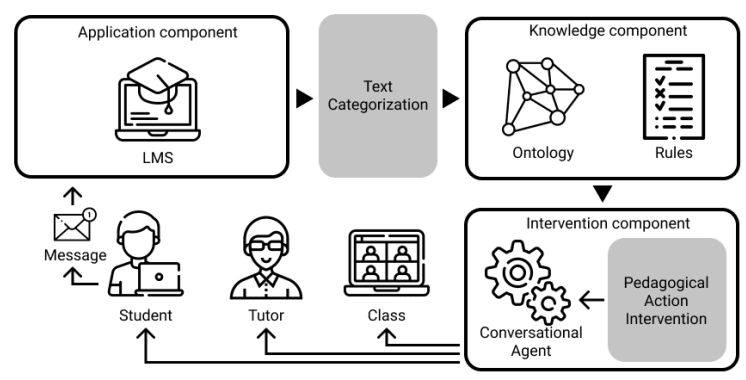

Figure 1. Framework proposal with its three main components.

\subsection{Knowledge Component}

The post data and its attributes must be stored, making it possible to understand the context and its related entities. An ontology was developed to meet the needs above as a way of organizing information that allows the agent to make inferences automatically. Thus, it is possible to discover implicit information, regardless of the volume of data. The ontology classes, presented in Figure 2, that were identified in the proposed context are:

- Message: responsible for storing the text with the student's post;

- Area: stores the area of knowledge that the student's course is related to;

- Urgency: defines the degree of urgency that the message needs to be attended to, originally in [Capuano and Caballé 2019], the values vary from 1 to 7 to represent this characteristic, 1 being less urgent and 7 more urgent. In this work, we will adopt whether the message is urgent or not;

- Confusion: determines how confusing the student's post is, originally [Bóbó et al. 2019] the values could range from 1 to 7, where 1 is the least level of confusion and 7 the highest level of confusion, but for the objective of this work we adopted whether there was or not confusion in the student's post, the values were also converted into confused and not confused;

- Sentiment: this property designates the sentiment passed through the student text. The values are negative, neutral, and positive [Bóbó et al. 2019][Demetriadis et al. 2018].

- Actor: receives the entities involved in the intervention process, where the actor can assume the role of tutor, student, agent, class;

- Type: this option stores the type of the message that the student sent. This message can be opinion, question and answer;

- Intervention: the kind of pedagogical mediation that was used to interfere in the learning process. It may be a motivational message, a help message, a thank message, help from the class, or tutor help. 


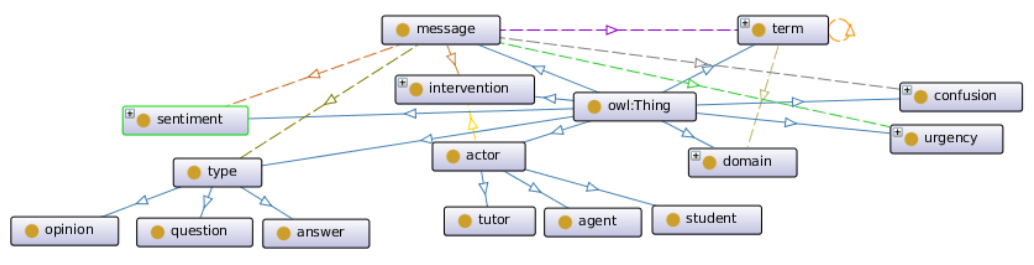

Figure 2. Post Categorization Ontology.

\subsection{Intervention Component}

A hierarchical diagram was developed to identify the actors involved and the pedagogical intervention process. This formal visual representation enabled the creation of semantic classes and rules. Figure 3 shows part of the diagram, which represents the student's post of type opinion. Due to lack of space, the question and answer types were not presented in this paper. Based on the ontology classes, we identified the actions that the agent can take. So, the conversation agent provides as output:

1. Agent motivational message:Upon detecting an opinion that requires this type of intervention, the conversational agent selects a motivational message from the available resources.

2. Agent help:According to the post, the conversational agent takes action by directly helping the author, trying to clear up the existing confusion.

3. Agent thank message:In this intervention process, the conversational agent sends the author of the post a thank you for the contribution. This intervention is detected when there is no negative feeling, urgency, and confusion.

4. Agent class help:When there is no urgency to answer the student's post, but there is confusion, in an attempt to encourage interaction through peers, the agent asks students in the same class to try to help the author of the post.

5. Tutor Help:The most critical cases that deserve greater attention are sent to the tutor responsible for the MOOC so that the intervention process can be efficient.

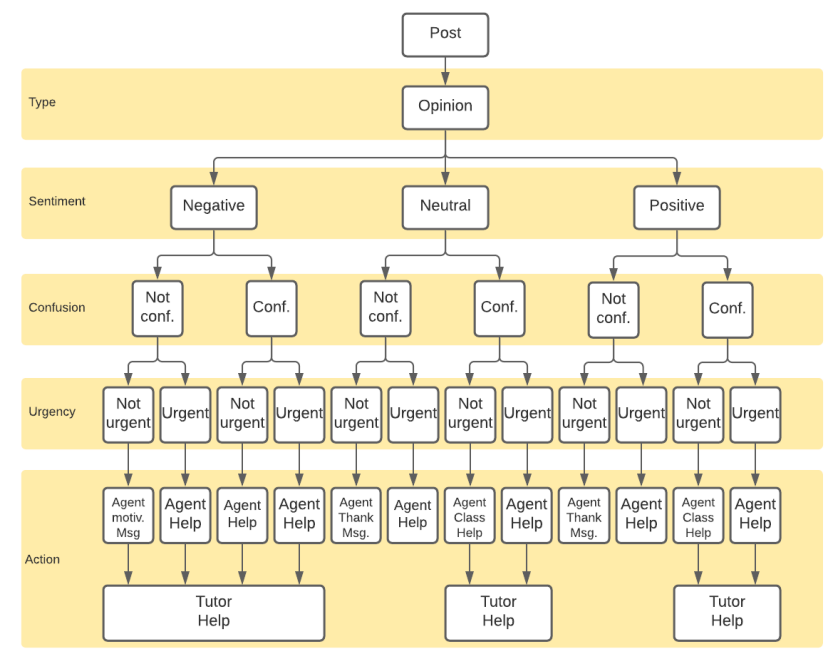

Figure 3. Hierarchical Diagram with the Opinion post formal representation.

The data from the students' posts are loaded into the ontological structure, allowing the information to be consistently related and inferences are made. To accurately 
determine the pedagogical intervention process that the agent should use, it is necessary to apply semantic rules, which guide us in choosing the pedagogical intervention process, taking into account the attributes related to the post. For constructing the ontology, the Protégé tool was used together with the OWL language, as it is a semantic markup language that allows describing the classes and their relationships. We loaded the data by using the Java language together with the OWL API, which allowed filling the instances after reading the posts and their data in the dataset. Table 1 presents some rules in SWRL. It is important to emphasize that only a few samples of rules are being presented.

Table 1. SWRL rules to detect pedagogical intervention.

\begin{tabular}{|c|c|}
\hline Rule & Description \\
\hline $\begin{array}{l}\text { message(?a)^express(?a, ?b)^swrlb:equal(?b, "neg- } \\
\text { ative")^isConnected(?a, ?c)^swrlb:equal(?c, "opin- } \\
\text { ion")^ intervention(?d)^swrlb:equal(?d, "Tutor } \\
\text { help") - require(?a, ?d) }\end{array}$ & $\begin{array}{l}\text { There is a relationship with the } \\
\text { intervention "Tutor help" for } \\
\text { all posts of the opinion and } \\
\text { negative feeling type. }\end{array}$ \\
\hline $\begin{array}{l}\text { message(?a)^express(?a, ?b)^swrlb:equal(?b, "neg- } \\
\text { ative")^ isConnected(?a, ?c)^swrlb:equal(?c, "opin- } \\
\text { ion") ^ describe(?a, ?d)^swrlb:equal(?d, "Not ur- } \\
\text { gent") ^ report(?a, ?e)^swrlb:equal(?e, "Not con- } \\
\text { fused")^ intervention(?f)^ swrlb:equal(?f, "Agent } \\
\text { motivational message") - require(?a,?f) }\end{array}$ & $\begin{array}{l}\text { For posts of the negative opin- } \\
\text { ion and feeling type, which are } \\
\text { not urgent and have no con- } \\
\text { fusion, an intervention of the } \\
\text { type "Agent motivational mes- } \\
\text { sage" is designated. }\end{array}$ \\
\hline $\begin{array}{l}\text { message(?a)^isConnected(?a, ?c)^swrlb:equal(?c, } \\
\text { "opinion")^ describe(?a, ?d)^swrlb:equal(?d, "Ur- } \\
\text { gent")^ intervention(?f) ^ swrlb:equal(?f, "Agent } \\
\text { help") - require(?a, ?f) }\end{array}$ & $\begin{array}{l}\text { It is related to the "Agent help" } \\
\text { type intervention all opinions } \\
\text { that have urgency. }\end{array}$ \\
\hline $\begin{array}{l}\text { message(?a)^ } \operatorname{express}(? \mathrm{a}, ? \mathrm{~b}) \wedge \text { swrlb:notEqual(?b, } \\
\text { "negative")^ isConnected(?a, ?c)^swrlb:equal(?c, } \\
\text { "opinion")^ describe(?a, ?d)^swrlb:equal(?d, "Not } \\
\text { urgent")^ report(?a, ?e)^swrlb:equal(?e, "Not con- } \\
\text { fused")^ intervention(?f)^ swrlb:equal(?f, "Agent } \\
\text { thank message") - require(?a, ?f) }\end{array}$ & $\begin{array}{l}\text { Opinions that do not have neg- } \\
\text { ative sentiment, do not require } \\
\text { urgency, and do not express } \\
\text { confusion are linked to the } \\
\text { "Agent thank message" inter- } \\
\text { vention type. }\end{array}$ \\
\hline 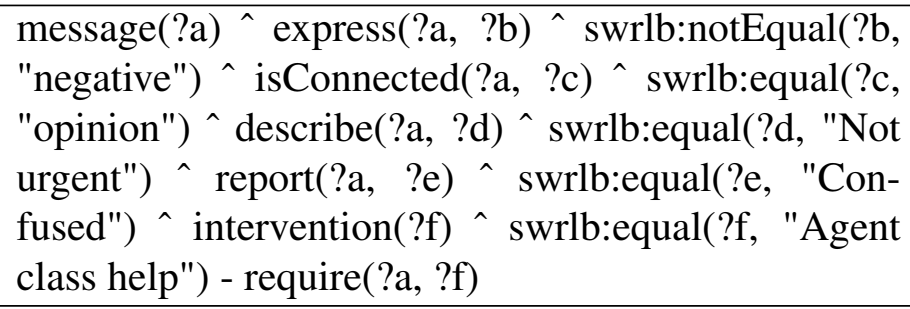 & $\begin{array}{l}\text { All opinions that do not ex- } \\
\text { press a negative sentiment and } \\
\text { are not urgent but have confu- } \\
\text { sion are linked to an interven- } \\
\text { tion of the "Agent class help" } \\
\text { type. }\end{array}$ \\
\hline
\end{tabular}

\section{Feasibility Study}

In this section, we conduct a feasibility study, presenting real use case scenarios in which the framework can be used to load posts into the ontology and determine the appropriate actions and interventions based on the identified rules. A feasibility study attempts to characterize a technology to ensure that it does what it claims to do and is worth developing [dos Santos 2016]. As this is the first effort to implement the solution, it has become prohibitive to apply more traditional assessment methods, such 
as case studies. So, instead, we use the Goal-Question-Metric (GQM) methodology [Caldiera and Rombach 1994].

The purpose of this evaluation is: analyze the framework with the purpose of evaluating with respect to its ontological rules from the point of view of professors and tutors in the context of providing useful pedagogical interventions. We thereby derive the following questions: Q1) Can the framework post categorization ontology be used to provide semantic rules for the conversational agent? Q2) Is the solution able to guide the agent to identify pedagogical interventions to help professors and tutors in MOOCs environments?

\subsection{Validating the ontological rules}

The ontology was loaded with the posts from Stanford MOOCPosts dataset ${ }^{1}$, a real dataset containing 29.604 anonymized messages, referring to eleven Stanford online courses. This dataset has already been used by other similar works[Agrawal et al. 2015] [Capuano and Caballé 2019] [Rossi et al. 2021]. These courses are concentrated in three knowledge areas, and the collection of posts was carried out for 14 months. For the data to be loaded into the ontology, it was necessary to perform a pre-processing step, where some data needed to be converted to the expected standard. Originally, the dataset has the post type divided into three attributes, opinion (yes/no), question (yes/no), and answer (yes/no), so it was necessary to check which attribute has the answer "yes" and convert it for the post type.

The sentiment attribute also needed a conversion, as the values ranged from 1 to 7 , being 1 the most negative sentiment and 7 the most positive one. As the framework works with negative, neutral, and positive sentiments, posts with values between 1 and 3 were labeled with negative sentiment. Those with values from 3.5 to 4.5 were labeled with the neutral sentiment, and posts with values higher than 5 were tagged with positive sentiment. For the attributes confusion and urgency, values from 1 to 7 were converted to the presence or not of these characteristics. Therefore, values up to 4 represent posts without the attribute, and from 5 to 7, we have posts characterized as confusion and urgency.

In the dataset, we found 16.469 (55.6\%) posts representing the students' opinions. We created six semantic rules to obtain the pedagogical intervention for these type of posts. Some of them were selected as a sample and are presented to show the necessary interventions. Table 2 summarizes the results of this step, presenting the percentage of data considering the ontology class "Type".

\subsection{Identifying the pedagogical intervention}

In this evaluation step, considering the rules activated by the ontology in relation to the student's post, we analyzed the agent action and the pedagogical intervention. It is possible to see in Table 2 that $44.7 \%$ of the messages are classified as positive or neutral, not confusing, and not urgent. Thus, according to the identified rules, the conversational agent acts autonomously, automatically answers to the post, preventing the tutor from having to waste time to answer them.

On the other hand, some messages need immediate intervention, and, in these cases, the tutor or professor must analyze them specifically. Figure 4 shows a sample

\footnotetext{
${ }^{1}$ https://datastage.stanford.edu/StanfordMoocPosts/
} 
Table 2. Quantitative of posts grouped taking attributes into account.

\begin{tabular}{|l|l|l|l|}
\hline Sentiment & Urgency & Confusion & Total \\
\hline Negative & Not urgent & Not confused & $431(2,62 \%)$ \\
\hline Negative & Not urgent & Confused & $472(2,87 \%)$ \\
\hline Negative & Urgent & Not confused & $11(0,07 \%)$ \\
\hline Negative & Urgent & Confused & $325(1,97 \%)$ \\
\hline Neutral & Not urgent & Not confused & $5349(32,48 \%)$ \\
\hline Neutral & Not urgent & Confused & $5122(31,10 \%)$ \\
\hline Neutral & Urgent & Not confused & $146(0,89 \%)$ \\
\hline Neutral & Urgent & Confused & $1457(8,85 \%)$ \\
\hline Positive & Not urgent & Not confused & $2018(12,25 \%)$ \\
\hline Positive & Not urgent & Confused & $985(5,98 \%)$ \\
\hline Positive & Urgent & Not confused & $26(0,16 \%)$ \\
\hline Positive & Urgent & Confused & $127(0,77 \%)$ \\
\hline
\end{tabular}

of students' posts, the conversational agent's action, and the pedagogical intervention. When the student sent the message, it was processed by the ontology, and the agent took action. As it is a negative sentiment message, the conversational agent sent a motivational message in the class forum and asked for tutor help in a private chat. In this way, the student knows that something was done concerning his message.

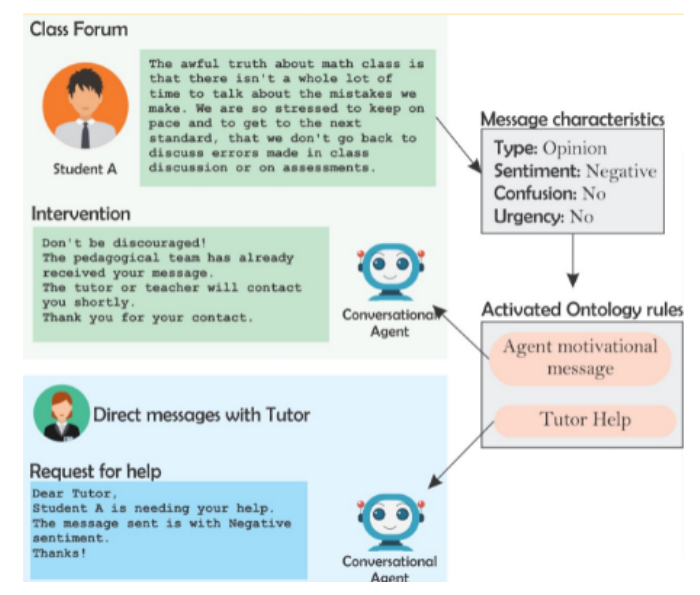

Figure 4. Sample of one of the negative messages sent by a student in the MOOC and the conversational agent actions.

The messages sent by the conversational agent may vary according to the identified pedagogical intervention process. Therefore, some standard messages are:

1. Agent help and Tutor help: Hilary, I noticed that you need help urgently, so I've already notified the pedagogical team. The tutor will contact you shortly. In the meantime, can I offer help to [Concept1]? Thanks for your contact.

2. Agent thank message: James, your participation was crucial. Dedication and studies are the secrets to success. So make as much contribution as possible. It makes our interaction environment richer. Thank you for your contact.

3. Agent help: Oliver, I realized you need help. Do you want to get help for [Concept1]? 
4. Agent class help and Tutor help: Do you agree or disagree with what was said by Chloe? I would like classmates to help us with this task. The tutor was also invited to this conversation. Thanks for listening.

In summary, 25,399 interventions were generated for the dataset posts, among them, $431(1.7 \%)$ were of the agent motivational message type, 2,564 (10.1\%) are agent help, 7,367 (29.0\%) are agent thank message, 6,107 (24.0\%) are agent class message and $8,930(35.2 \%)$ are tutor help. According to the interventions inferred through the rules, it is possible to notice that the tutor's effort is significantly reduced.

\subsection{Discussion}

After the ontology was developed, it was possible to load the posts and their data and the links between them. The dataset has 29,604 posts from 11,043 different students, therefore all messages were stored. By loading the post into the ontology, we can make inferences based on the previously defined rules. Thus, the necessary pedagogical intervention is identified, and the conversational agent can make decisions and adopt an approach more suited to the student's needs. As the student/tutor ratio is very high, it is not always possible to respond immediately to all posts in the forums, which can negatively affect the student, making them feel abandoned and often insecure for not being closely monitored by a person able to help them. Thus, the agent's performance can significantly reduce the number of messages that need attention, leaving only the most critical posts for the tutor. It helps the tutor to be more agile and act better in serving this student; in addition, no student remains unanswered, so we greatly reduce the possibility of the students feeling abandoned by the tutor. We can understand that it is possible to answer the research questions, as the first question seeks to verify if ontology can provide semantic rules for the agent. According to the numbers obtained after loading the data, it is possible to conclude that the ontology provides the rules. The second question investigates the solution's ability to help the tutor in MOOC environments, as shown above, in addition to identifying the necessary pedagogical intervention, only about $35 \%$ of the posts need the tutor's attention.

\section{Final Remarks}

In this article, we build an ontology with a set of semantic rules, capable of guiding the conversational agent, indicating the type of pedagogical intervention. This approach can contribute a lot to the quality of teaching, through this help in interactions through student posts, mainly in MOOC's environments, where the tutor has a huge difficulty to keep up with all the students, therefore, it also contributes to the tutor, a since automatic interventions can happen automatically. Some limitations were observed in the development of the work, such as classifying new posts, that is when we do not have the attributes to describe the type, feeling, confusion and urgency. In future work, we intend to resolve these limitations by performing the automatic classification of posts. This can contribute to expanding posts beyond those present in Stanford MOOC Posts datasets.

\section{Acknowledgment}

This work was carried out with the support of the Coordination of Improvement of Higher Education Personnel - Brazil (CAPES) - Financing Code 001, Federal University of Juiz de Fora (UFJF), CNPq (435313/2018-5) and FAPEMIG. 


\section{References}

Agrawal, A., Venkatraman, J., Leonard, S., and Paepcke, A. (2015). Youedu: addressing confusion in mooc discussion forums by recommending instructional video clips.

Bóbó, M., Campos, F., Stroele, V., David, J., and Braga, R. (2019). Identificação do perfil emocional do aluno através de análise de sentimento: Combatendo a evasão escolar. In Brazilian Symposium on Computers in Education (Simpósio Brasileiro de Informática na Educação-SBIE), volume 30, page 1431.

Braz, F., Campos, F., Stroele, V., and Dantas, M. (2019). An early warning model for school dropout: A case study in e-learning class. In Brazilian Symposium on Computers in Education (Simpósio Brasileiro de Informática na Educação-SBIE), volume 30, page 1441.

Brigui-Chtioui, I., Caillou, P., and Negre, E. (2017). Intelligent digital learning: Agentbased recommender system. In Proceedings of the 9th International Conference on Machine Learning and Computing, pages 71-76.

Caldiera, V. R. B.-G. and Rombach, H. D. (1994). Goal question metric paradigm. Encyclopedia of software engineering, 1:528-532.

Callaghan, M. J., Bengloan, G., Ferrer, J., Cherel, L., El Mostadi, M. A., Eguíluz, A. G., and McShane, N. (2018). Voice driven virtual assistant tutor in virtual reality for electronic engineering remote laboratories. In International Conference on Remote Engineering and Virtual Instrumentation, pages 570-580. Springer.

Capuano, N. and Caballé, S. (2015). Towards adaptive peer assessment for moocs. In 2015 10th International Conference on P2P, Parallel, Grid, Cloud and Internet Computing (3PGCIC), pages 64-69. IEEE.

Capuano, N. and Caballé, S. (2019). Multi-attribute categorization of mooc forum posts and applications to conversational agents. In International Conference on P2P, Parallel, Grid, Cloud and Internet Computing, pages 505-514. Springer.

Demetriadis, S., Caballé, S., Papadopoulos, P. M., Gómez-Sánchez, E., Kolling, A., Tegos, S., Tsiatsos, T., Psathas, G., Michos, K., Weinberger, A., et al. (2021). Conversational agents in moocs: reflections on first outcomes of the colmooc project.

Demetriadis, S., Tegos, S., Psathas, G., Tsiatsos, T., Weinberger, A., Caballé, S., Dimitriadis, Y., Sánchez, E. G., Papadopoulos, P. M., and Karakostas, A. (2018). Conversational agents as group-teacher interaction mediators in moocs. In 2018 Learning With MOOCS (LWMOOCS), pages 43-46. IEEE.

dos Santos, R. P. (2016). Managing and monitoring software ecosystem to support demand and solution analysis. PhD thesis, Universidade Federal do Rio de Janeiro.

Harrathi, M., Touzani, N., and Braham, R. (2017). A hybrid knowlegde-based approach for recommending massive learning activities. In 2017 IEEE/ACS 14th International Conference on Computer Systems and Applications (AICCSA), pages 49-54. IEEE.

Neves, F., Campos, F., Ströele, V., Capretz, M. A., Jennings, M., Bryant, D., and Dantas, M. (2020). Heath-prior: An intelligent ensemble architecture to identify risk cases in healthcare. IEEE Access, 8:217150-217168. 
Rossi, D., Ströele, V., Braga, R., Caballé, S., Capuano, N., Campos, F., Dantas, M., Lomasto, L., and Toti, D. (2021). Caers: A conversational agent for intervention in moocs' learning process. In The learning ideas conference.

Toti, D., Capuano, N., Campos, F., Dantas, M., Neves, F., and Caballé, S. (2020). Detection of student engagement in e-learning systems based on semantic analysis and machine learning. In International Conference on P2P, Parallel, Grid, Cloud and Internet Computing, pages 211-223. Springer. 